\title{
HIV and Depression: 2008 Review and Update
}

\author{
Judith G. Rabkin, PhD, MPH
}

\section{Corresponding author}

Judith G. Rabkin, PhD, MPH

Department of Psychiatry, College of Physicians and Surgeons, Columbia University, 1051 Riverside Drive, Unit 51, New York, NY 10032, USA.

E-mail: jgr1@columbia.edu

Current HIV/AIDS Reports 2008, 5:163-171

Current Medicine Group LLC ISSN 1548-3568

Copyright (C) 2008 by Current Medicine Group LLC

Since the earliest days of the AIDS epidemic, clinicians have been concerned about the prevalence of depression among their patients. Epidemiologists, psychiatrists, psychologists, sociologists, and a broad array of other specialists have studied this topic, trying to determine the prevalence of depressive disorders and depressive symptoms as well as antecedents, correlates, and consequences. This review addresses the methodologic difficulties in determining depression prevalence, major findings regarding rates of disorder and correlates among different segments of the HIV community, effects of depression on HIV illness progression, psychopharmacologic and psychotherapeutic treatment findings, and behavioral effects of depression, such as its impact on medication adherence, employment, and risk behavior. Finally, the article summarizes international studies of depression prevalence in developing countries and the challenges regarding cross-national diagnostic definitions and measures.

\section{Introduction}

In the context of HIV/AIDS, depression is an often overlooked but potentially dangerous condition that can influence not only quality of life, relationships, employment, and adherence to medical care, but also perhaps survival. Depression is associated with isolated lives, the absence of pleasure, and social and vocational impairment. Depression is also associated with failure to maintain a proper diet and exercise regimen and to adhere to medical care. Other than substance use disorders, depression is the most prevalent psychiatric disorder among $\mathrm{HIV}$-positive $(\mathrm{HIV+})$ adults. It can be identified even in primary care settings once the topic is raised (which is insufficiently often), and its treatment is one of psychiatry's strengths.

This brief review covers several complex areas. Space limitations preclude comprehensive reviews of any topic; instead, representative studies are cited, with an emphasis on recent literature. This article does not include studies of depression in HIV+ youth or reports from the literature on substance use disorders.

\section{Prevalence and Correlates of Depression in HIV/AIDS}

Although depression is common, determining its prevalence across studies and within different segments of the HIV population is not easy, given the variety of methodologic issues that warrant consideration. A review of salient issues helps in reconciling disparate or inconsistent findings in the literature.

\section{Methodologic issues}

Variation in choice of criteria for diagnosis, of measures, and of respondent samples complicates comparison of findings across studies, and precludes easy synthesis of findings. Definitions of depression have changed over time: since the first prevalence studies published in the 1980s, investigators have successively used the diagnostic criteria of the Diagnostic and Statistical Manual of Mental Disorders (DSM)-III (1980) [1], DSM-IIIR (1987) [2], and DSM-IV (1994) [3]. International studies use International Classification of Diseases (ICD)-9 or ICD-10 criteria (see "Appendices" in DSM-IV). More problematic than these slightly different criteria are variations in assessment methods, ranging from "gold standard" structured psychiatric interviews administered by professional mental health investigators, to diagnoses based on clinician-administered psychiatric "screeners," to self-report symptom checklists, virtually none of which were designed to generate diagnoses. The choice of measurement tool is often related to sample size: in national and international samples including hundreds of respondents, it is not usually feasible for clinicians to conduct face-to-face, detailed psychiatric interviews; therefore, screeners administered by trained lay interviewers are the next best choice. However, many studies with a broader psychosocial focus rely exclusively 
on self-report symptom scales that are sensitive but nonspecific, and thus are likely to be elevated and assess distress rather than syndromal depression. Commonly used selfreport scales, all of which are described in Rush et al. [4], include the Center for Epidemiologic Studies-Depression Scale (CES-D), Symptom Checklist 90 (SCL-90) or Brief Symptom Inventory (a shorter version of the SCL-90), or the Beck Depression Inventory. More recently, the Patient Health Questionnaire (PHQ-9) has become available. The PHQ-9, which is the only self-report scale intended to generate "approximate" diagnoses, consists of the nine criteria for major depression with a response format inquiring about frequency of occurrence and presence of behavioral impairment secondary to depressive symptoms, following DSM-IV diagnostic rules.

Even when the same or very similar diagnostic methods are employed, investigators often vary in the number of diagnoses they include to determine depression prevalence. Although major depressive disorder is always included, some investigators also include dysthymia, a more chronic and often less severe form of depression. Less commonly included are subthreshold major depression (four of the nine criteria required for major depression), minor depression (three of the nine criteria), adjustment disorder with depressed mood, and depression "not otherwise specified," a residual category that lacks specificity entirely.

Another major source of variation is sample selection. When samples include population subgroups with high rates of depression independent of HIV infection, such as injection drug users, the rates are elevated [5•]. Recruitment based on clinic samples tends to yield higher rates of depression than studies with community volunteers. Moreover, HIV illness stage may vary among samples. This is particularly problematic in samples of late-stage patients in whom symptom checklists are used; somatic symptoms such as fatigue, insomnia, and weight loss may be attributable to depression, HIV illness, or concurrent medications for HIV or comorbid conditions such as hepatitis C. Another consideration in cross-sample comparison is the evolving population of those with HIV/AIDS. Early research in the 1980s and early 1990s focused on middle-class white homosexual men, who were then the predominant group identified as $\mathrm{HIV+}$. In recent years, however, heterosexual men and women increasingly have been represented in prevalence studies, more often coming from disadvantaged communities with background elevations in depression prevalence independent of HIV status. More recent studies have included larger samples, although some have lacked control groups, and in many cases have relied on self-report symptom checklists.

Finally, time frame, age range, and gender vary among studies. Some studies inquire about symptoms in the past 2 weeks, the past month, the past 6 months, the past year, or the patient's lifetime, including current symptoms. The longer the time frame, the higher the rate of depression.
In the general population, age is also associated with variation in depression rates, with younger cohorts having higher rates of current and lifetime depression than respondents older than 50 or 55 years; differences are also seen among socioeconomic groups and between the sexes, with higher depression rates seen in low-income groups and women [6].

\section{Prevalence rates}

Numerous studies have been conducted with widely varying samples, measures, and results; therefore, a synthesis of findings is elusive. Earlier reviews of studies of prevalence of major depression in $\mathrm{HIV}+$ respondents have found rates ranging from $1 \%$ to $48 \%$ [7,8]. Studies published before the advent of combination antiretroviral therapy varied in sample size, usually focused on homosexual men, used structured psychiatric interviews, and often included comparison groups of HIV-seronegative respondents from the same communities. Overall rates of major depressive disorder were in the $5 \%$ to $10 \%$ range. Within studies, no differences due to HIV serostatus were found [9], although in a meta-analysis, rates were significantly higher for the HIV+ respondents [10].

The first national study of the prevalence of psychopathology in HIV+ respondents-the HIV Cost and Services Utilization Study (HCSUS)—used a diagnostic screener to assess 2864 adults receiving care for HIV; a subset of these patients then underwent a full diagnostic interview. Based on the screener, $36 \%$ of the patients tested positive for major depression and $27 \%$ for dysthymia [11]. Re-estimation based on the full diagnostic interview resulted in a $22 \%$ prevalence rate for major depression and 5\% for dysthymia [12]. In addition to determining depression rates in a national sample, this study exemplifies the difference in prevalence as a function of the diagnostic method used.

Recent studies have found varying rates of depression. A retrospective analysis of more than $3300 \mathrm{HIV+}$ patients-predominantly white homosexual men-in managed care systems found that $42 \%$ had a diagnosis of depression in their medical records on at least one outpatient visit; however, there is no information regarding whether the depression was transient or persistent, or how the diagnoses were defined and made [13]. Other studies have found high rates in HIV+ and HIV-negative (HIV-) male and female injection drug users, unrelated to HIV serostatus (eg, [14,15]).

In HIV samples, as in general population studies, depression rates for women are elevated, although in some studies this is true independent of HIV serostatus. Moore et al. [16], in a multisite study of $871 \mathrm{HIV}+$ women and 439 demographically matched HIV-seronegative women in economically and socially disadvantaged environments, found that both groups reported high levels of adverse events and depressive symptoms unrelated to HIV status. In contrast, Morrison et al. [17] found rates of major 
depression among HIV+ women $(19 \%)$ to be four times greater than the rate among HIV-seronegative women (5\%); Evans et al. [18], studying 93 women from disadvantaged backgrounds, also found higher rates of major depression among HIV+ women (15\% vs $10 \%)$.

One of the diagnoses of exclusion for depression is bipolar disorder, based on occurrence of past or current manic episodes, in which case the diagnosis is bipolar disorder. In the context of HIV/AIDS, descriptive and treatment studies are lacking. No large diagnostic surveys of HIV+ respondents have included bipolar disorder (eg, [11,19]), and no treatment studies of HIV+ bipolar disorder have been conducted. Primary and secondary mania has been described in HIV-infected patients, although chart studies and case series were reported in the 1990s [20]. Mania that predates HIV infection may be a risk factor for HIV transmission because impulsivity, disinhibition, hypersexuality, and elevated use of drugs and alcohol are likely to interfere with safer sex practices. In addition, new-onset or secondary mania has been described among patients with more advanced immunosuppression. This is thought to be associated with HIV central nervous system involvement, is sometimes associated with structural brain damage demonstrable by CT or MRI [21], and often occurs with cognitive decline [22,23]. Clinicians, as well as investigators who screen out patients with bipolar disorders from their studies, have noted that they see more HIV+ bipolar patients than expected (eg, Bangsberg, personal communication). Differential diagnosis of bipolar disorder versus unipolar depression is important because treating a bipolar patient with antidepressants alone may trigger a manic episode. Bipolar disorder is best treated by psychiatrists, whereas unipolar depression is often successfully managed by HIV care providers.

Comorbidity of psychiatric disorders is common in both the general and HIV populations. A substantial number of people who are clinically depressed have another diagnosis at the same time. This was illustrated in a study of $152 \mathrm{HIV+}$ patients consecutively presenting at an academic medical center. The patients in this southeastern clinic were primarily 30 to 50 years old, about one third were female, and $75 \%$ were African American. Structured clinical interviews for DSMIV axis I/II disorders (SCIDs) included mood, anxiety, and substance use disorders and were administered by phone or in person [24]. Twenty-one percent of the patients were diagnosed with a current (within the past month) mood disorder, $17 \%$ with an anxiety disorder (posttraumatic stress disorder [PTSD]), and $11 \%$ with a substance use disorder (crack cocaine or alcohol). Of patients with at least one disorder in the past year, half had two or more diagnoses and of these, $40 \%$ had current (past month) diagnoses. Of the 32 patients with current depression, 9 also had PTSD, 3 also had a substance use diagnosis, and 5 had all three disorders. In this series, older age, fewer HIV-related medical symptoms, and use of antiretroviral therapy were each associated with lower prevalence of any psychiatric disorder.
Another condition comorbid with psychiatric disorders is cognitive impairment, which was seen more often in the earlier days of the HIV epidemic. Although there is no causal association, the presence of both makes clinical management and treatment more challenging. In an innercity HIV clinic in Baltimore, almost $20 \%$ of patients had significant cognitive impairment [25].

Although, overall, rates of depressive disorders among $\mathrm{HIV}+$ adults may or may not be elevated compared with HIV- persons from the same community, they clearly are greater than the rates for the general population. In a nationally representative study, 9090 community respondents in 48 states were selected to reflect the 2000 census [6]. Using face-to-face household surveys and structured psychiatric interviews, the investigators found a 12-month prevalence of major depressive disorder of $16 \%$ for life and $6.6 \%$ for the past 12 months. As an approximate overall estimate, Evans et al. [26] proposed a range of 5\% to $20 \%$ for current major depression among HIV+ adults, depending on the sample. In addition, depressive symptoms that do not meet criteria for disorder are relatively common. At the same time, it should be emphasized that most people with HIV/AIDS are not depressed most of the time, and their resilience is as noteworthy as their psychopathology.

\section{Depression, HIV Stage, and Mortality}

By themselves, immunologic and virologic markers of HIV progression unrelated to medical symptoms are not systematically related to rates of depression in cross-sectional studies (eg, [27,28]). Results from longitudinal cohort studies of depression are less consistent. Although some investigators failed to find such an association [29-32], others did find one (eg, [33]).

Although medically symptomatic HIV+ patients often report higher rates of depression compared with asymptomatic HIV+ or HIV- comparison groups, as noted it requires effort to distinguish between somatic symptoms of depression, such as weight loss and fatigue, and concurrent medical symptoms. A recently published study with a 2-year prospective design and semiannual assessments, including HIV+ and HIV- men, found that incident major depressive episodes were more likely among $\mathrm{HIV}+$ men with symptomatic disease than among HIV- men or asymptomatic HIV+ men [34].

A related question is whether depression is a predictor of clinical progression of HIV/AIDS. In the earliest study, conducted before the advent of antiretroviral combination therapy, 395 homosexual men in the San Francisco Men's Study, which began in 1984, were followed up for 9 years [35]. Men with symptoms of depression on the CES-D self-report scale at study entry (defined as scores $\geq 16$ ) had a higher risk of progression to AIDS; the relative hazard was 1.4. In the Carolina Cohort Study, severe life stress increased the risk of early disease progression [36]. Bouhnik et al. [37] also found that depression 
predicted clinical progression (decline in CD4 cells or death) in a sample of $305 \mathrm{HIV}+$ drug users being treated with antiretroviral medication. This effect was independent of nonadherence behaviors.

Two large cohort studies found an association between chronic depression and mortality. Ickovics et al. [38] observed $765 \mathrm{HIV}+$ women over 7 years and found significant associations between depressive symptoms and HIV progression and mortality; women with chronic depressive symptoms on the CES-D were twice as likely to die as women with minimal or absent symptoms. In the Women's Interagency HIV Study (WIHS) [39], 1716 urban women at six sites were assessed semiannually over a 7-year period using the self-report CES-D scale. Two fifths of the women reported a history of substance use, and 39\% did so during the study. About one third reported depressive symptoms (CES-D scores $>16$ ) at $75 \%$ or more of their clinic visits, and nearly half did so at their last study visit. Chronic depressive symptoms predicted mortality; $13 \%$ died from AIDS-related causes, compared with $6 \%$ or $7 \%$ of women with minimal or intermittent depressive symptoms. However, it remains unclear whether the primary reasons for this association involve poorer adherence to medical care and medications or biological mechanisms mediating disease acceleration, as discussed by Kopnisky et al [40].

Leserman [41], in a comprehensive literature review, concluded that chronic depression, trauma, and stressful events together may negatively affect HIV disease progression in terms of CD4 decline and increases in viral load, and pose greater risk for clinical decline. Most of the evidence predates the widespread use of antiretroviral combination therapy. Whether access to and utilization of medical care is a covariate has not been clarified. It also remains to be determined "whether depression puts HIV infected persons at greater risk for disease progression, or whether changes in disease may be associated with increased risk of depression" [41] (p. 542).

\section{Risk factors}

As noted by Atkinson et al. [34], populations at highest risk for HIV infection have a high prevalence of primary (preexisting) mood disorders; therefore, elevated rates observed after HIV infection may reflect new episodes of preexisting disorders rather than new-onset depression. Elevated rates of depressive disorders have been reported among people with same-sex behavior [42-44] and substance use disorders $[11,45]$. Other risk factors include a history of domestic conflict and violence, bereavement, lower social status and history of substance abuse [46], and family history of depression. As is true of the general population, HIV+ women have higher rates of depressive symptoms than HIV+ men [47].

Because, until recently, HIV/AIDS was a disease of youth and middle age, few of the earlier prevalence studies of psychopathology included enough HIV+ older adults to examine separately; even today, older is defined as "over age 49 " in most HIV studies of aging. It is estimated that over $25 \%$ of all people with AIDS in the United States are older than 50 years, and the proportion in New York City is $30 \%$ [48]. In all major population studies, rates of depressive disorders decrease substantially by age and birth cohort. For example, in a nationally representative population study of 9000 community respondents, 12month and lifetime rates for the youngest cohort (ages 18-29 years) are nearly double the rates for the oldest cohort (age $\geq 60$ years) [6]. In another survey of 43,000 adults, current rates of major depression declined by nearly $50 \%$ between the same age groups [49]. Overall, the decline with age is dramatic, not subtle, in community samples. In HIV+ samples, a similar pattern was found, with lower rates of depression reported by those older than 50 years in a nationally representative study by Bing et al. [11] and a three-site Veterans Aging Cohort study [50]. On the other hand, in their five-site Veterans Aging Cohort study, Justice et al. [51] found that depressive symptoms did not decline with age among HIV+ veterans. Rabkin et al. [52] compared small samples of HIV+ and HIV- men younger and older than 50 years and found that although HIV- men over 50 had lower rates of depression than younger HIV- men, as in the general population, there was no decline among the HIV+ men over 50. Karpiak et al. [48] found that one third of 914 inner-city HIV+ adults over 50 had high scores on the CES-D, a self-report rating scale. Only the study by Justice et al. [51] included an HIV-seronegative comparison group of older adults; in this study, depression rates did decline with age. Overall, the population trend is for lower rates of depression with increasing age; the available evidence for HIV+ adults suggests that this decline with age may not occur in older adults with HIV, although an increase in older HIV+ adults relative to younger groups has not been reported.

\section{Correlates}

To summarize and expand on the foregoing studies, correlates of current depression include a history of depression, acute symptomatic illness (as is true in studies of patients with other serious medical illnesses [26]), substance abuse, younger age, female gender, perceptions of HIVrelated stigma $[53,54]$, and social isolation $[7,55]$.

\section{Treating Depression in the Context of HIV/AIDS}

In the 1980s, when AIDS was an invariably fatal disease with rapid progression after diagnosis, it was unclear whether depression was "only to be expected" or was, in fact, a treatable disorder, as it is in medically healthy patients. However, double-blind placebo-controlled trials and open-label studies consistently have demonstrated that HIV+ patients who are depressed respond in the same proportion as other patients, and that depression is indeed a treatable condition in the 
context of HIV/AIDS (see Ferrando and Wapenyi [56] for a review of this early literature). Antidepressant medications shown to be effective in treating HIV+ depressed patients include imipramine, sertraline, fluoxetine, nefazodone, citalopram, bupropion, mirtazapine, and venlafaxine. Some studies found larger effects than did others, but all showed some efficacy. Recently, Ferrando and Freyberg [57•] provided a comprehensive review of depression treatment, and Repetto and Petitto [58] published a more general review including other psychiatric disorders as well as depression.

Depression treatment studies have included a range of participants from different backgrounds, although most exclude patients who are current drug abusers. The rationale is that it is difficult to evaluate the effect of a study medication on mood if the patient is independently using a mood-altering substance at the same time. Also, the majority of participants in most studies have been male, although a few small studies targeted women [59]. Bangsberg et al. [60] tackled a particularly difficult population, evaluating directly observed therapy (DOT) with long-acting fluoxetine compared with referral to psychiatric care in a sample of homeless or marginally housed HIV+ adults, including active substance users, in San Francisco. After the first 2 weeks of daily DOT, medication was switched to long-acting fluoxetine, with augmenting medications permitted. Control group members visited the study site weekly to equalize exposure to study staff and were asked whether they were taking antidepressants. Adherence was high, attrition modest, and results impressive: both groups had improved mood, but the DOT group had fewer depressive symptoms at every 3 -month data point over 36 weeks, and twice as many continued to take antidepressants throughout the period of observation at week 36 .

Nontraditional treatments for depression, such as testosterone [61], dehydroepiandrosterone [62], and dextroamphetamine [63], also have been found to be effective in placebo-controlled studies. For patients with both fatigue and depression, modafinil reduced depressive symptoms in an open-label trial [64].

Safety concerns have been raised regarding potential interactions of antidepressants and antiretroviral medications because they share similar metabolic pathways. The concern that drug interactions conceivably could slow or hasten metabolism of antiretrovirals has not been documented $[65,66]$. The likelihood of drug interactions is greatest with the protease inhibitors, which are less widely used and are prescribed at lower doses as newer antiretrovirals in different classes become available.

Research on the efficacy of psychotherapy for depressed HIV+ patients is extremely limited. As Ferrando and Freyberg [57•] noted, only one study compared individual psychotherapies with antidepressant medication for HIV+ patients with depressive symptoms [67]. In this study, interpersonal psychotherapy and imipramine plus supportive psychotherapy were found to be more effective than cognitive behavioral therapy or supportive psychotherapy alone.
In another design, group psychotherapy with or without fluoxetine was assessed, with good responses in both conditions $[68,69]$. Other studies were designed to reduce stress levels, improve coping, and promote behavioral changes such as improved medication adherence; however, they did not select for baseline depression and are not antidepressant trials [70,71].

Overall, treatment of depression in patients with HIV/ AIDS is as effective as it is in medically healthy patients. Choice of treatment should be based on considerations such as accessibility (eg, interpersonal psychotherapy, a manualized therapy, is not widely available), side effects profile (eg, mirtazapine is not indicated for a patient with concomitant fatigue), and past experience with effective or ineffective treatments. The most notable problem in managing depression in the context of HIV/AIDS is its identification: too few care providers routinely ask patients if they are depressed, and too few patients volunteer the information spontaneously.

\section{Behavioral Effects of Depression Medication adherence}

Because adherence to antiretroviral medications is central to their effectiveness in preventing disease progression and extending survival, characteristics of patients with poor medication adherence have been studied extensively over the past 12 years. Depressed mood is often associated with nonadherence, although there is not yet prospective evidence that treatment of depression improves medication adherence among patients with self-reported or documented medication nonadherence. The available evidence consists of chart and pharmacy record reviews. Horberg et al [13] reviewed charts of 3359 patients in two health maintenance organizations; $42 \%$ of the patients were identified as depressed and $15 \%$ were using antidepressants during antiretroviral therapy. Depression was associated with poorer medication adherence and HIV viral control. However, depressed patients who took their antidepressant medication (defined by pharmacy refills) had viral control similar to that of nondepressed patients. The report by Horberg et al. [13] also summarizes prior studies showing an association between depression and medication nonadherence. Comparable findings have been observed in other chart reviews [72]. A recently funded depression treatment study for patients identified as nonadherent to HIV medications is currently under way and may be the first to provide direct evidence of the association of depression and medication adherence (Wagner, personal communication).

\section{Employment and return to work}

Depression is widely recognized as a major cause of impaired work performance, missed work days, and general disease burden; unipolar depression is expected to be the second leading cause of disease burden (measured by disability- 
adjusted life years) in the next decade, exceeded only by ischemic heart disease [73]. In the context of HIV/AIDS, there are multiple reasons to leave work, as well as barriers to re-employment. Although the major determinants are probably health insurance and disability benefits, depression also plays a role both in the decision to stop work and as a barrier to returning to work once health is stable. In a longitudinal study of 141 men with AIDS followed up on six semiannual occasions, the major predictors of unemployment or partial employment, in order of influence, were financial considerations (disability benefits), psychiatric factors (past or current diagnosis of major depression or dysthymia), medical status, cognitive (neuropsychological) factors, and education [74]. Overall, most men unemployed at study entry did not return to work, despite stabilized health. In another longitudinal study, 118 predominantly symptomatic HIV+ men and women who had actually initiated steps to return to work at study entry were followed up over 2 years [75]. Over time, $40 \%$ of the participants got jobs of at least 5 hours/week for at least 1 week. However, only $15 \%$ worked persistently once employed. Memory function was the best predictor of finding work. Depressive symptoms were not a predictor of participants' return to work, but scores on the Beck Depression Inventory declined significantly after they found work. Other studies of HIV+ adults and employment have found comparably low success rates $(13 \%-16 \%)$ in returning to permanent paid employment $[76,77]$. Finally, 38 HIV+ adults seeking treatment for depression who expressed a desire to return to work were evaluated before starting antidepressant medication and an average of 5 months later (McElhiney et al., unpublished data). Only five patients had actually found steady work of 15 hours/week or more, and another three worked a few hours a week on a temporary basis. Those who returned to work did not differ from those who did not in terms of baseline sociodemographic, neuropsychological, or medical characteristics. However, although both groups improved, those who returned to work had lower depression scores and reported more peer support for working. This small literature suggests a role for depression in unemployment for HIV+ adults who are medically able to work, although depression treatment alone appears to be insufficient in promoting return to work.

\section{Depression and sexual risk behavior}

In general, diminished libido is associated with depressive disorders among both men and women [78]. In fact, in an earlier version of the current standard criteria for diagnosing mental disorders, "loss of interest in or enjoyment of sex" was one of the nine criteria used to diagnose depression (DSM-III, page 220 [1]), although it did not appear 7 years later in DSM-IIIR (revised edition) [2]. However, research findings regarding sexual risk behavior in depressed HIV+ adults have been inconsistent; some studies have found lower levels of risk, whereas others have reported increased risk behavior in states of depression, as reviewed by Bradley et al. [79]. Whether risk behavior, or indeed any sexual behavior, increases in depressed HIV+ adults with low libido whose depression is successfully treated has not been systematically investigated. In the early 1990s, ethical questions were raised about a study in which men with AIDS, low libido, and low serum testosterone levels were treated with intramuscular testosterone [80], on the theory that any "increase in sexual desire" among HIV-infected men presents a public health risk [81]. However, another commentator disagreed [82], and the authors noted that it is the obligation of health providers to offer treatment for a medical deficiency state (hypogonadism) that has been diagnosed. The same argument would apply to treatment of depression. Although alleviation of depression is likely to restore libido if it is diminished during depression, another likely effect is to offset the hopelessness and nihilism that can underlie risky sexual behavior.

\section{International Studies}

A surge of interest in international HIV studies has produced dozens of research projects in African countries and elsewhere, although until recently most efforts have been devoted to research addressing counseling and testing, risk reduction and prevention research, and medication adherence. Major questions arise concerning definition of psychiatric syndromes across cultures [83], measurement issues, and issues of informed consent. Understanding the meaning of observed prevalence rates of disorders in subgroups (eg, those with HIV/AIDS) requires appropriate comparison groups or information about rates in the background population, neither of which may be available in many resource-poor settings.

The first and largest international study of mood and anxiety disorders was conducted in 1990 to 1991 by Maj et al. [19] in five centers: Zaire, Kenya, Thailand, Germany, and Brazil. At each site, structured diagnostic interviews were administered to samples of HIV+ asymptomatic, HIV+ symptomatic, and HIV-seronegative men and women recruited from outpatient clinics in the same medical setting. Sites varied by composition; for example, homosexual men were the predominant group in Brazil, whereas in Bangkok nearly all participants were intravenous drug users. Overall, this study demonstrated the feasibility of comparative international research using standardized Western measures on prevalence of psychiatric disorders. In brief summary, symptomatic HIV+ participants had higher rates of depression than HIV-seronegative respondents in all sites. Rates of current major depression varied by country (and its sample composition) such that depression was least prevalent in Germany, Zaire, and Kenya across serostatus groups $(<6 \%$ in any subgroup) and the highest rates $(14 \%-24 \%)$ were reported in Thailand. 
Collins et al. [84] reviewed 30 studies assessing depression in HIV+ respondents in Africa, Asia, Eastern Europe, and Central and South America. The measures, diagnostic categories included, and samples were heterogeneous, as were the findings: rates of depression ranged from $0 \%$ to $63 \%$. In Uganda, Musisi et al. [85] conducted several studies of depression in different populations, ranging from $\mathrm{HIV}+$ orphaned adolescents to residents of the war zone of northern Uganda and patients coinfected with HIV and tuberculosis. In Ethiopia, several large-scale epidemiologic research programs have been undertaken under the leadership of Atalay Alem, MD, among both stable and semi-nomadic populations, using the Composite International Diagnostic Interview (CIDI; also used by Maj et al. [19]) as the diagnostic instrument [86]. One program compared the CIDI and key informants in a methodologic case-finding study, showing the CIDI to be superior in identifying those with major depression [87]. Another investigated sociodemographic correlates of major depression in rural Ethiopia, based on a door-to-door survey of 70,000 residents, with older age and status as formerly married associated with risk for depression [88]. These programs show that such research is feasible, even in difficult field situations with a dearth of clinicians trained in psychiatry and in respondents unfamiliar with psychiatric concepts. Although they do not easily lend themselves to summary, these studies illustrate that Western diagnostic measures in translation appear to be valid, and that depression is present in meaningful proportions in most HIV+ populations globally, even if treatment options are not.

\section{Conclusions}

The enormous variation in reported prevalence rates by location, time frame, measures, diagnostic category, and sample composition makes it impractical to arrive at a single estimate of depression prevalence associated with HIV/AIDS. However, we can conclude that, to interpret the meaning of any observed prevalence estimate, comparison groups or populations are essential to understand what is associated with HIV and what is not, which subgroups within the widely varied HIV population are being considered, and what are the lifetime prevalence rates of disorder. After substance abuse, depression is the most common psychiatric disorder in HIV populations; the behavioral implications for personal and community health are significant, and depression is as treatable in $\mathrm{HIV}+$ patients as in the general population, with equal success rates.

\section{Disclosure}

No potential conflict of interest relevant to this article was reported.

\section{References and Recommended Reading}

Papers of particular interest, published recently, have been highlighted as:

- Of importance

- Of major importance

1. Diagnostic and Statistical Manual of Mental Disorders, edn 3. (DSM-III). Washington, DC: American Psychiatric Association; 1980.

2. Diagnostic and Statistical Manual of Mental Disorders, edn 3-revised (DSM-IIIR). Washington, DC: American Psychiatric Association; 1987.

3. Diagnostic and Statistical Manual of Mental Disorders $(D S M-I V)$. Washington, DC: American Psychiatric Association; 1994.

4. Rush J, First M, Blacker D, eds: Handbook of Psychiatric Measures, edn 2. Washington, DC: American Psychiatric Publishing ; 2008.

5. Chander G, Himelhoch S, Moore R: Substance abuse and psychiatric disorder in HIV-positive patients. Drugs 2006, 66:769-789.

Extensive review with 164 references, focusing on substance abuse prevalence and its effect on medication adherence, adherence interventions for HIV+ individuals with concurrent substance use disorders, and depression, severe mental illness, and anxiety disorders and their prevalence in HIV+ samples.

6. Kessler R, Berglund P, Demler O, et al.: The epidemiology of major depressive disorder: results from the National Comorbidity Survey Replication (NCS-R). JAMA 2003, 289:3095-3105.

7. Stober D, Schwartz J, McDaniel S, et al.: Depression and HIV disease: prevalence, correlates and treatment. Psychiatr Ann 1997, 27:372-377.

8. Lyketsos C, Federman E: Psychiatric disorders and HIV infection: impact on one another. Epidemiol Rev 1995, 17:152-164.

9. Rabkin JG: Prevalence of psychiatric disorders in HIV illness. Int Rev Psychiatry 1996, 8:157-166.

10. Ciesla JA, Roberts J: Meta-analysis of the relationship between HIV infection and risk for depressive disorders. Am J Psychiatry 2001, 158:725-730.

11. Bing EG, Burnam M, Longshore D, et al.: Psychiatric disorders and drug use among human immunodeficiency virus-infected adults in the United States. Arch Gen Psychiatry 2001, 58:721-728.

12. Orlando M, Burnam M, Beckman R, et al.: Re-estimating the prevalence of psychiatric disorders in a nationally representative sample of persons receiving care for HIV: results from the HIV Cost and Services Utilization Study. Int J Methods Psychiatr Res 2002, 11:75-82.

13. Horberg MA, Silverberg M, Hurley L, et al.: Effects of depression and selective serotonin reuptake inhibitor use on adherence to highly active antiretroviral therapy and on clinical outcomes in HIV-infected patients. J Acquir Immune Defic Syndr 2008, 47:384-390.

14. Rabkin JG, Johnson J, Lin S, et al.: Psychopathology in male and female HIV-positive and negative injecting drug users: longitudinal course over 3 years. AIDS 1997 , 11:507-515.

15. Knowlton A, Latkin C, Chung S, et al.: HIV and depressive symptoms among low-income illicit drug users. AIDS Behav 2000, 4:353-360.

16. Moore J, Schuman P, Schoenbaum E, et al.: Severe adverse events and depressive symptoms among women with, or at risk for, HIV infection in four cities in the United States of America. AIDS 1999, 13:2459-2468.

17. Morrison MF, Petitto J, Ten Have T, et al.: Depressive and anxiety disorders in women with HIV infection. $A m \mathrm{~J}$ Psychiatry 2002, 159:789-796. 
18. Evans D, Ten Have T, Douglas S, et al.: Association of depression with viral load, CD8 T lymphocytes, and natural killer cells in women with HIV infection. Am J Psychiatry 2002, 159:1752-1759.

19. Maj M, Janssen R, Starace F, et al.: WHO neuropsychiatric AIDS study, cross-sectional phase I: study design and psychiatric findings. Arch Gen Psychiatry 1994, 51:39-49.

20. Lyketsos C, Hanson A, Fishman M, et al.: Manic syndrome early and late in the course of HIV. Am J Psychiatry 1993, 150:326-327.

21. Mijch A, Judd F, Lyketsos C, et al.: Secondary mania in patients with HIV infection. J Neuropsychiatry Clin Neurosci 1999, 11:475-480.

22. Lyketsos C, Schwartz J, Fishman M, et al.: AIDS mania. J Neuropsychiatry Clin Neurosci 1997, 9:277-279.

23. Nakimuli-Mpungu E, Musisi S, Mpungu S, Katabira E: Primary mania vs. HIV-related secondary mania in Uganda. Am J Psychiatry 2006, 163:1349-1354.

24. Gaynes B, Pence B, Eron J, et al.: Prevalence and comorbidity of psychiatric diagnoses based on reference standard in an HIV+ patient population. Psychosom Med 2008, 70:505-511.

25. Treisman G, Angelino A, Hutton H: Psychiatric issues in the management of patients with HIV infection. JAMA 2001, 286:2857-2864.

26. Evans DL, Charney D, Lewis L, et al.: Mood disorders in the medically ill: scientific review and recommendations. Biol Psychiatry 2005, 58:175-189.

27. Atkinson JH, Grant I, Kennedy C, et al.: Prevalence of psychiatric disorders among men infected with HIV virus: a controlled study. Arch Gen Psychiatry 1988, 45:859-864.

28. Rabkin JG, Ferrando SJ, Jacobsberg LB, Fishman B: Prevalence of axis I disorders in an AIDS cohort: a cross-sectional, controlled study. Compr Psychiatry 1997, 38:146-154.

29. Rabkin JG, Goetz R, Remien R, et al.: Stability of mood despite HIV illness progression in a group of homosexual men. Am J Psychiatry 1997, 154:231-238.

30. Rabkin JG, Williams JBW, Remien R, et al.: Depression, distress, lymphocyte subsets and HIV symptoms on two occasions in HIV+ male homosexuals. Arch Gen Psychiatry 1991, 48:111-119.

31. Lyketsos CG, Hoover D, Guccione M, et al.: Depressive symptoms as predictors of medical outcomes in HIV infection. JAMA 1993, 270:2563-2567.

32. Burack J, Barrett D, Stall R, et al.: Depressive symptoms and CD4 lymphocyte decline among HIV-infected men. JAMA 1993, 270:2568-2573.

33. Mayne T, Vittinghoff E, Chesney M, et al.: Depressive affect and survival among gay and bisexual men infected with HIV. Arch Intern Med 1996, 156:2233-2238.

34. Atkinson H, Heaton R, Patterson T, et al.: Two-year prospective study of major depressive disorder in $\mathrm{HIV}+$ men. J Affect Disord 2007 108:225-234.

35. Page-Shafer K, Delorenze G, Satariano W, Winkelstein W: Comorbidity and survival in HIV-infected men in the San Francisco Men's Health Survey. Ann Epidemiol 1996, 6:420-430.

36. Evans D, Leserman J, Perkins D, et al.: Severe life stress as a predictor of early disease progression in HIV infection. Am J Psychiatry 1997, 154:630-634.

37. Bouhnik A, Preau M, Vincent E, et al.: Depression and clinical progression in HIV-infected drug users treated with highly active antiretroviral therapy. Antivir Ther 2005 , 10:53-61.

38. Ickovics J, Hamburger M, Vlahov D, et al.: Mortality, CD4 cell count decline, and depressive symptoms among HIV seropositive women. JAMA 2001, 285:1466-1474.

39. Cook JA, Grey D, Burke J, et al.: Depressive symptoms and AIDS-related mortality among a multisite cohort of HIV+ women. Am J Public Health 2004, 94:1133-1140.

40. Kopnisky K, Stoff D, Rausch D: Workshop report: the effects of psychological variables on the progression of HIV-1 disease. Brain Behav Immun 2004, 18:246-261.
41. Leserman J: Role of depression, stress, and trauma in HIV disease progression. Psychosom Med 2008, 70:539-545.

42. Sandfort T, de Graaf R, Bijl R, et al.: Same-sex sexual behavior and psychiatric disorders: findings from the Netherlands Mental Health Survey and Incidence Study (NEMESIS). Arch Gen Psychiatry 2001, 58:85-91.

43. Cochran SD, Mays V: Depressive distress among homosexually active African American men and women. $\mathrm{Am} \mathrm{J}$ Psychiatry 1994, 151:524-529.

44. Mills TC, Paul J, Stall R, et al.: Distress and depression in men who have sex with men: the Urban Men's Health Study. Am J Psychiatry 2004, 161:278-285.

45. Rabkin JG, Johnson J, Lin S, et al.: Psychopathology in male and female HIV-positive and negative injecting drug users: longitudinal course over 3 years. AIDS 1997, 11:507-515.

46. Leserman J: The effects of stressful life events, coping and cortisol on HIV infection. CNS Spectr 2003, 8:25-30.

47. Valverdee EE, Purcell D, Waldrop-Valverfe D, et al.: Correlates of depression among HIV-positive women and men who inject drugs. J Acquir Immune Defic Syndr 2007, 46(Suppl 2):S96-S100

48. Karpiak SE, Shippy RA, Cantor MH: Research on older adults with HIV. New York: AIDS Community Research Initiative of America (ACRIA). Available at http://www. acria.org/clinical/roah_05_05_08_final.pdf. Accessed May 23, 2008.

49. Hasin D, Goodwin R, Stinson F, et al.: Epidemiology of major depressive disorder. Results from the National Epidemiologic Survey on Alcoholism and Related Conditions. Arch Gen Psychiatry 2005, 62:1097-1106.

50. Zingmond D, Kilbourne A, Justice A, et al.: Differences in symptom expression in older HIV-positive patients: the Veterans Aging Cohort 3 Site Study and HIV Cost and Service Utilization Study experience. J Acquir Immune Defic Syndr 2003, 33:S84-S92.

51. Justice A, McGinnis K, Atkinson J, et al.: Psychiatric and neurocognitive disorders among HIV-positive and negative veterans in care: Veterans Aging Cohort Five-Site Study. AIDS 2004, 18(Suppl 1):S49-S59.

52. Rabkin JG, McElhiney M, Ferrando SJ: Mood and substance use disorders in older adults with HIV/AIDS: methodological issues and preliminary evidence. AIDS 2004, 18(Suppl 1): S43-S48.

53. Emlet CA: An examination of the social networks and social isolation in older and younger adults living with HIV/AIDS. Health Soc Work 2006, 31:299-308.

54. Flowers P, Davis M, Hart G, et al.: Diagnosis and stigma amongst HIV positive Black Africans living in the UK. Psychol Health 2006, 21:109-122.

55. Catz SL, Gore-Felton C, McClure J: Psychological distress among minority and low-income women living with HIV. Behav Med 2002, 28:53-60.

56. Ferrando SJ, Wapenyi K: Psychopharmacological treatment of patients with HIV and AIDS. Psychiatr Q 2002, 73:33-49.

57. $\quad$ Ferrando SJ, Freyberg Z: Treatment of depression in HIV-positive individuals: an evidence-based review. Int Rev Psychiatry 2008, 20:61-71.

This comprehensive review, with 41 references and two tables, includes both pharmacologic and psychotherapeutic interventions for depression in HIV-positive patients.

58. Repetto MJ, Petitto J: Psychopharmacology in HIV-infected patients. Psychosom Med 2008, 70:585-592.

59. Ferrando SJ, Rabkin J, deMoore G, Rabkin R: Antidepressant treatment of depression in HIV-seropositive women. J Clin Psychiatry 1999, 60:741-746.

60. Bangsberg DR, Hammer G, Karasic D, et al.: Directly observed weekly fluoxetine improves depression symptom severity in HIV+ homeless and marginally housed depressed individuals. Presented at the 2 nd International Conference on Adherence to HIV Antiretroviral Therapy. Jersey City, NJ; March 20, 2006. 
61. Rabkin JG, Wagner G, Rabkin R: A double-blind, placebocontrolled trial of testosterone therapy for HIV-positive men with hypogonadal symptoms. Arch Gen Psychiatry 2000, 57:141-147.

62. Rabkin JG, McElhiney M, Rabkin R, et al.: Placebocontrolled trial of dehydroepiandrosterone (DHEA) for treatment of nonmajor depression in patients with HIV/AIDS. Am J Psychiatry 2006, 163:1-8.

63. Wagner G, Rabkin R: Effects of dextroamphetamine on depression and fatigue in HIV+ men: a double-blind, placebo controlled trial. J Clin Psychiatry 2000, 61:436-440.

64. Rabkin JG, McElhiney M, Rabkin R, Ferrando SJ: Modafinil treatment of fatigue in HIV+ patients: a pilot study. J Clin Psychiatry 2004, 65:1688-1695.

65. De Maat MM, Huitema A, Mulder J, et al.: Drug interaction of fluvoxamine and fluoxetine with nevirapine in HIV-1infected individuals. Clin Drug Invest 2003, 23:629-637.

66. Farber EW, McDaniel JS: Clinical management of psychiatric disorders in patients with HIV disease. Psychiatr Q 2002, 73:5-16.

67. Markowitz J, Kocsis J, Fishman B, et al.: Treatment of depressive symptoms in HIV-positive patients. Arch Gen Psychiatry 1998, 55:452-457.

68. Targ EF, Karasic D, Diefenbach P, et al.: Structured group therapy and fluoxetine to treat depression in HIV-positive persons. Psychosomatics 1994, 35:132-137.

69. Zisook S, Peterkin J, Goggin K, et al.: Treatment of major depression in HIV-seropositive men. J Clin Psychiatry 1998, 59:217-224.

70. Laperriere A, Ironson G, Antoni M, et al.: Decreased depression up to one year following CBSM+ intervention in depressed women with AIDS: the Smart/EST women's project. J Health Psychol 2005, 10:223-231.

71. Carrico A, Antoni M, Duran R, et al.: Reductions in depressed mood and denial coping during cognitive behavioral stress management with HIV-positive gay men treated with HAART. Ann Behav Med 2006, 31:155-164.

72. Yun L, Maravi M, Kobayashi J, et al.: Antidepressant treatment improves adherence to antiretroviral therapy among depressed HIV-infected patients. J Acquir Immune Defic Syndr 2005, 38:432-438.

73. Murray C, Lopez A, eds.: The Global Burden of Disease: Summary. Cambridge, MA: Harvard University Press; 1996.

74. Rabkin JG, McElhiney M, Ferrando S, et al.: Predictors of employment of men with HIV/AIDS: a longitudinal study. Psychosom Med 2004, 66:72-78.
75. Van Gorp W, Rabkin J, Ferrando S, et al.: Neuropsychiatric predictors of return to work in HIV/AIDS. J Int Neuropsychol Soc 2006, 13:80-89.

76. Goldman DP, Baio Y: Effective HIV treatment and employment of HIV+ adults. Health Serv Res 2004, 39:1691-1712.

77. Lem M, Moore D, Marion S, et al.: Back to work: correlates of employment among persons receiving highly active antiretroviral therapy. AIDS Care 2005, 17:740-746.

78. Williams K, Reynolds M: Sexual dysfunction in major depression. CNS Spectr 2006, 11:8(Suppl 9):19-23.

79. Bradley M, Remien R, Dolezal C: Depression symptoms and sexual HIV risk behavior among serodiscordant couples. Psychosom Med 2008, 70:186-191.

80. Wagner G, Rabkin J, Rabkin R: Effects of testosterone replacement on sexual interest, function and behavior in HIV+ men. J Sex Res 1997, 34:27-33; 37-38.

81. Fisher W: Do no harm: on the ethics of testosterone replacement therapy for HIV+ persons. J Sex Res 1997, 34:35-36.

82. Tiefer L: Response to testosterone injection study and Fisher's concerns. J Sex Res 1997, 34:37.

83. Wilk CM, Bolton P: Local perceptions of the mental health effects of the Uganda AIDS epidemic. J Nerv Ment Dis 2002, 190:394-397.

84. Collins PY, Holman A, Freeman M, Patel V: What is the relevance of mental health to HIV/AIDS care and treatment programs in developing countries? A systematic review [editorial review]. AIDS 2006, 20:1571-1582.

85. Musisi S, Nakasujja N, Zziwa J: A comparison of depression among patients with HIV/AIDS and pulmonary tuberculosis. Proceedings of the African Association of Psychiatry and Allied Professions Annual Conference. Nairobi, Kenya; March 21-23, 2007.

86. Alem A: Stanley Medical Research Institute Funded Studies of Schizophrenia and Bipolar Disorders in Ethiopia 1997-2006 Report. Addis Ababa, Ethiopia: Addis Ababa University Printing Press; 2007.

87. Shibre T, Kebede D, Alem A, et al.: An evaluation of two screening methods to identify cases with schizophrenia and affective disorders in a community survey in rural Ethiopia. Int J Soc Psychiatry 2002, 48:200-208.

88. Kebede D, Alem A, Deyassa N, et al.: Socio-demographic correlates of depressive disorder in Butajira, rural Ethiopia. Cent Afr J Med 2003, 49:78-83. 